\title{
Cirurgia valvar em crianças e jovens: resultados de 131 casos
}

Luís Roberto GEROLA*, Pablo M. A. POMERANTZEFF*, Paulo M. PÊGO-FERNANDES*, Noedir A. G. STOLF*, Miguel BARBERO-MARCIAL*, Munir EBAID*, Rachel SNITCOWSKY*, Max GRINBERG*, Geraldo VERGINELLI*, Adib D. JATENE*.

RBCCV 44205-124

GEROLA, L. R.; POMERANTZEFF, P. M. A.; PÊGo-FERNANDES, P. M.; STOLF, N. A. G.; BARBEROMARCIAL, M.; EBAID, M.; SNITCOWSKY, R.; GRINBERG, M.; VERGINELLI, G.; JATENE, A. D. Cirurgia valvar em crianças e jovens: resultados de 131 casos. Rev. Bras. Cir. Cardiovasc., 5(3): $187-194,1990$.

RESUMO: No período de janeiro de 1983 a dezembro de 1988,131 pacientes, com idade igual ou inferior a 15 anos, foram submetidos a cirurgia valvar, $74(56 \%)$ de etiologia adquirida e $57(44 \%)$ de etiologia congênita. Em $100(76,3 \%)$ pacientes somente uma valva foi tratada. Foram realizadas 63 trocas valvares (retroca em seis), 103 procedimentos conservadores e três exéreses valvares. Das 63 próteses utilizadas, $59(93,6 \%)$ eram biológicas e $4(6,3 \%)$ mecânicas. Vinte e um $(16 \%)$ casos eram reoperaçōes, sendo $17(81 \%)$ por disfunção de prótese. O tempo médio de calcificação das biopróteses foi de 40 meses (3,3 anos). A mortalidade hospitalar foi de $7,5 \%$ e a tardia foi de $2,2 \%$. No seguimento tardio de 787 meses/paciente, todos encontram-se em classe funcional I e II (NYHA).

DESCRITORES: valvas cardíacas, cirurgia, crianças; valvas cardíacas, cirurgia, pacientes jovens.

\section{INTRODUÇÃO}

O número de pacientes jovens com lesōes valvares é considerável em nosso meio, sendo que a febre reumática ainda é a entidade causadora em um grande número destes.

O tratamento cirúrgico ideal para as valvopatias é aquele que corrige as lesōes anatômicas através de procedimentos conservadores ${ }^{5-8,36}$, possibilitando normalização hemodinâmica. Entretanto, as plásticas valvares, que acreditamos devem ser cada vez mais estimuladas, principalmente nesta faixa etária, nem sempre sāo possíveis, determinando o uso de próteses valvares.

A escolha da prótese a ser utilizada nunca é fácil e geralmente depende de muitos fatores, tais como o nível sócio-econômico do paciente, experiência do Serviço com determinada prótese, possibili- dade de anticoagulação e controle da mesma. As biopróteses em pacientes jovens têm durabilidade questionável " e as próteses mecânicas, cada vez mais utilizadas em jovens, ainda são dependentes da anticoagulaçăo e dos cuidados que este procedimento requer.

Neste momento, quando são discutidas as melhores condutas para correçāo das lesōes valvares em pacientes jovens, acreditamos ser oportuna a apresentação da nossa experiência, principalmente com as biopróteses.

\section{CASUISTICA E MÉTODOS}

Foram estudados 131 portadores de valvopatias, sendo $74(56,5 \%)$ adquiridas e $57(43,5 \%)$ congênitas, com idades inferiores a 15 anos, submetidos

\footnotetext{
Trabalho realizado no Instituto do Coração do Hospital das Clínicas da Faculdade de Medicina da Universidade de São Paulo. São Paulo, SP. Brasil. Recebido para publicaçáo em 10 de novembro de 1990.

* Do Instituto do Coraçáo do Hospital das Clínicas da Faculdade de Medicina da Universidade de Sảo Paulo.

Endereço: Luís Roberto Gerola. Av. Dr. Enéas de Carvalho Aguiar, 44. Divisão Cirúrgica. 05403 Sảo Paulo, SP. Brasil.
} 
GEROLA, L. R.; POMERANTZEFF, P. M. A.; PÊGO-FERNANDES, P. M.; STOLF, N. A. G.; BARBERO-MARCIAL, M.; EBAID, M.; SNITCOWSKY, R.; GRINBERG, M.; VERGINELLI, G.; JATENE, A. D. - Cirurgia valvar em crianças e jovens: resultados de 131 casos. Rev. Bras. Cir. Cardiovasc., 5(3): 187-194, 1990.

a tratamento cirúrgico. Setenta e quatro $(56,5 \%)$ eram do sexo masculino e $57(43,5)$ do feminino; as idades variaram de dois dias a 15 anos, com a média de 8,6 anos (Gráfico 1).

\section{GRÁFICO 1 \\ DISTRIBUIÇĀO DOS PACIENTES DE ACORDO COM A FAIXA ETÁRIA}

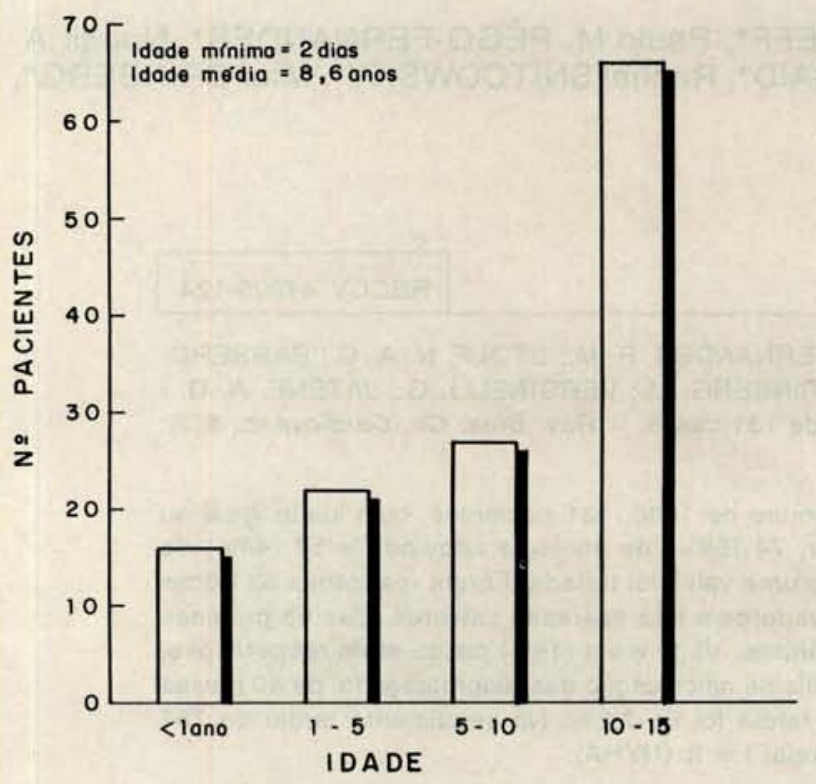

TABELA 1

DIAGNÓSTICOS PRINCIPAIS

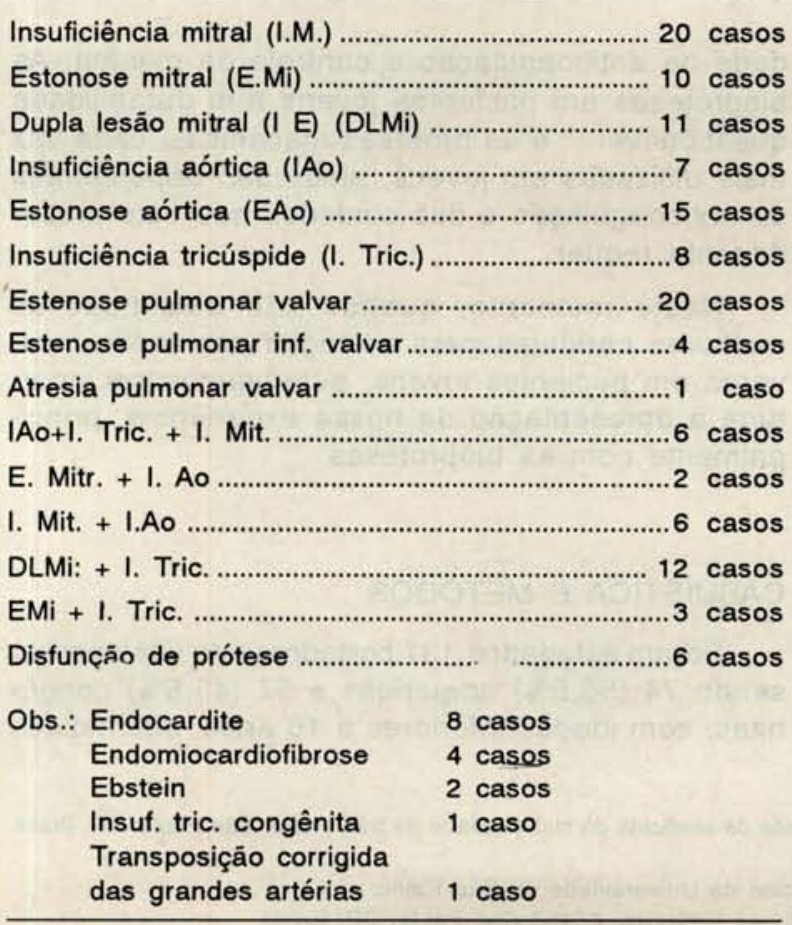

As lesōes valvares reumáticas mais freqüentes foram: insuficiência mitral ( 20 casos), estenose mitral (11 casos), dupla lesão mitral com predomínio de insuficiência (12 casos); as lesōes congênitas mais freqüentes foram: estenose aórtica (15 casos) e estenose pulmonar (20 casos) (Tabela 1).

A endocardite infecciosa foi diagnosticada em oito pacientes, em três na valva aórtica, em três na valva tricúspide e em dois nas valvas mitral e aórtica. $A$ endomiocardiopatia esteve presente em quatro pacientes, em uma valva tricúspide (VD) e em três nas valvas mitral e tricúspide (VE e VD).

Os três pacientes que apresentavam entenose mitral congênita tinham a valva em paraquedas e todas asssociadas a coartação da aorta. Dos portadores de insuficiência mitral congênita, em dois havia coartação da aorta e em um comunicação intraventricular. Nos doentes com lesão congênita da valva aórtica, a comunicação interventricular esteve presente em três casos, coartaçāo em um e persistência de canal arterial em outro.

Dos casos com lesão da valva tricúspide, dois correspondiam à doença de Ebstein, um apresentava insuficiência tricúspide congênita e outro transposiçăo corrigida dos grandes vasos da base.

Dos portadores de lesāo na valva pulmonar, drenagem anômala total das veias pulmonares esteve presente em um, doença de Ebstein em um e coartaçāo da aorta associada a persistência de canal arterial em outro.

Todos os pacientes foram avaliados clinicamente no pré-operatório segundo a NYHA, estando 19 $(14,5 \%)$ em classe funcional I, $38(29 \%)$ em classe funcional II, $35(26,8 \%)$ em classe funcional III e 39 $(29,7 \%)$ em classe funcional IV (Gráfico 2$)$.

Em $100(76 \%)$ pacientes somente um valva foi tratada, a mitral em 45 casos, a aórtica em 22, a pulmonar em 25 e a tricúspide em oito. Nos demais, mais de uma valva foi tratada; em 16 a mitral e a tricúspide, em oito a mitral e a aórtica e em sete a mitral, a aórtica e a tricúspide.

Nos 100 pacientes submetidos a operaçāo sobre uma valva, em $64(64 \%)$ foi possível sua conservaçāo, em $29(29 \%)$ foi necessária a troca valvar, retroca em $4(4 \%)$ e exérese valvar em três $(3 \%)$.

A valva mitral foi tratada isoladamente em 45 pacientes, $40(88,8 \%)$ de etiologia adquirida e 5 $(11,1 \%)$ congênita. A troca valvar foi realizada em $21(46,6 \%)$ pacientes, $19(90,5 \%)$ de etiologia adquirida e $2(9,5 \%)$ congênita; em $24(53,3 \%)$ foram realizados procedimentos conservadores, sendo 21 $(87,5 \%)$ de origem adquirida e $3(12,5 \%)$ congênita. Os principais procedimentos conservadores realizados foram: comissurotomia em três, comissurotomia 
GEROLA, L. R.; POMERANTZEFF, P. M. A.; PÊGO-FERNANDES, P. M.; STOLF, N. A. G.; BARBERO-MARCIAL, M.; EBAID, M.; SNITCOWSKY, R.; GRINBERG, M.; VERGINELLI, G.; JATENE, A. D. - Cirurgia valvar em crianças e jovens: resultados de 131 casos. Rev. Bras. Cir. Cardiovasc., 5(3): 187-194, 1990.

\section{GRÁFICO 2}

SITUAÇĀO CLÍNICA NO PRÉ E NO PÓS-OPERATÓRIO, DE ACORDO COM A NYHA. ÓBITOS IMEDIATOS (O.I.) E ÓBITOS TARDIOS. (O.T.)

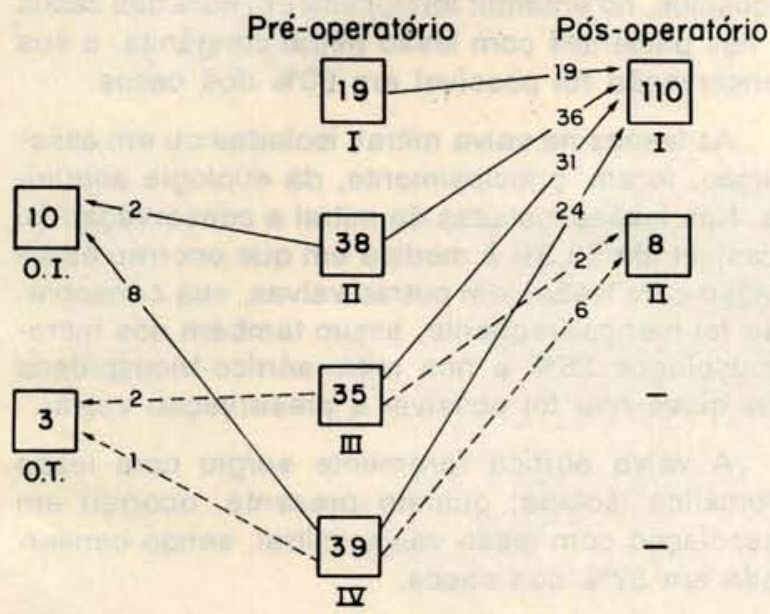

Tempo de seguimento $=2787$ meses $/$ pociente

e papilarotomia em 10 e plástica em 11, sendo nove anuloplastias, das quais quatro com anel de Carpentier e cinco com tira posterior (Teflon ou pericárdio); em um paciente foi realizada ampliação da cúspide posterior com pericárdio bovino e em outro, ressecção em cunha das cúspides anterior e posterior associada a anuloplastia posterior. Em cincos destes pacientes foi realizado encurtamento de corda tendínea.

A valva aórtica foi tratada isoladamente em 22 pacientes, $20(90,9 \%)$ com lesão congênita e $2(9,1 \%)$ com lesão adquirida em $6(27,2 \%)$ pacientes foi realizada a troca valvar, sendo $2(33,3 \%)$ substituiçōes em pacientes com lesão adquirida e $4(66,6 \%)$ em pacientes com lesōes congênitas. Em 16 (72,7\%) foi possível a conservação valvar, todos de etiologia congênita, sendo realizada comissurotomia em 12 , comissurotomia e ressecção de brotos embrionários em três e plástia em um (plicatura das cúspides).

A valva pulmonar foi tratada isoladamente em 25 pacientes, todos com lesão de etiologia congênita; em um foi realizada a exérese valvar, em 24 (96\%) foram realizados procedimentos conservadores, comissurotomia em 19 , comissurotomia e ressecção de infundíbulo em quatro e comissurotomia com ampliação do anel valvar em um.

A valva tricúspide foi tratada isoladamente em oito pacientes, metade de etiologia congênita; em $5(62,5 \%)$ foi realizada a troca valvar, dos quais 2 $(40 \%)$ de etiologia adquirida e $3(60 \%)$ congênita; em um paciente foi realizada plástica de De Vega (congênitos) e em dois exérese valvar (ambos por endocardite).
Nos pacientes submetidos a operação em mais de uma valva, todos apresentavam etiologia adquirida.

Nos 16 pacientes submetidos a operação nas valvas mitral e tricúspide, a mitral foi tratada em $11(68,1 \%)$ retrocada em $1(6 \%)$ e em $4(25 \%)$ foi conservada. A valva tricúspide foi conservada em todos os casos, sendo realizada plástica de De Vega.

Nos oito pacientes submetidos a operaçāo sobre as valvas aórtica e mitral, a amitral foi conservada em $7(87,5 \%)$ e trocada em $1(12,5 \%)$. A aórtica foi trocada em $5(62,5 \%)$ e conservada em $3(37,5 \%)$.

Nos sete pacientes submetidos a operação sobre as valvas mitral, tricúspide e aórtica, a aórtica foi conservada em $6(85,7 \%)$ e trocada em $1(14,2 \%)$, a mitral foi trocada em $6(85,7 \%)$ e retrocada em um $(14,2 \%)$ a valva tricúspide foi trocada em 5 $(71,4 \%)$ e retrocada em $2(28,5 \%)$.

\section{RESULTADOS}

Foram realizadas 63 trocas valvares (retroca em seis), 103 procedimentos conservadores e três exéreses valvares. (Tabela 2 ).

Das 63 próteses utilizadas, $59(93,6 \%)$ eram biológicas, 21 próteses Biocór (porcina), 25 EBM (pericárdio bovino) e sete Barone (porcina). Em 4 $(6,3 \%)$ pacientes foram utilizadas próteses mecâni-

TABELA 2

PROCEDIMENTOS REALIZADOS

\begin{tabular}{lcccc}
\hline & $\begin{array}{c}\text { Retroca } \\
\text { Valvar }\end{array}$ & $\begin{array}{c}\text { Troca } \\
\text { Valvar }\end{array}$ & $\begin{array}{c}\text { Proced. } \\
\text { Conservadores }\end{array}$ & $\begin{array}{c}\text { Exérese } \\
\text { Valvar }\end{array}$ \\
\hline Mitral & 4 & 17 & 24 & - \\
Aórtica & - & 6 & 16 & - \\
Tricúspide & - & 5 & 1 & 2 \\
Pulmonar & - & - & 24 & 1 \\
Mitral + Tricúspides & & & \\
MI & 1 & 11 & 4 & - \\
TRI & - & - & 16 & - \\
Mitral + Aórtica & - & 1 & 7 & - \\
MI & - & 5 & 3 & - \\
Ao & & 5 & & \\
Mitral + Aórtica + Tricúspide & 6 & - & - \\
MI & 1 & 6 & 2 & - \\
TRI & - & 5 & 6 & 3 \\
Ao & - & 1 & 103 & \\
\hline Total & 6 & 57 & & \\
\hline & & & & \\
\hline
\end{tabular}


GEROLA, L. R.; POMERANTZEFF, P. M. A.; PÊGO-FERNANDES, P. M.; STOLF, N. A. G.; BARBERO-MARCIAL, M.; EBAID, M.; SNITCOWSKY, R.; GRINBERG, M.; VERGINELLI, G.; JATENE, A. D. - Cirurgia valvar em crianças e jovens: resultados de 131 casos. Rev. Bras. Cir. Cardiovasc., 5(3): 187-194, 1990.

cas (duas Omnicarbon e duas Sorin) todas em posição aórtica.

As principais complicaçōes no pós-operatório imediato foram baixo débito em 21 (16\%) pacientes, coagulopatia em $15(11,4 \%)$ e arritmia em $6(4,5 \%)$. Houve quatro reoperaçōes, duas por tamponamento e duas por sangramento cirúrgico.

A mortalidade hospitalar foi de 7,5\% (10 pacientes), cinco portadores de cardiopatia congênita e cinco de cardiopatia adquirida, sendo um após reoperação (retroca mitral e plástica de De Vega).

Nos congênitos, o óbito foi conseqüênte a hipoxemia, broncoespasmo e baixo débito persistente (comissurotomia pulmonar em três, valvectomia em um e insuficiência tricúspide em um).

Nos adquiridos, o óbito foi conseqüente a baixo débito em três, embolia pulmonar em um e sangramento abdominal por lesāo do ligamento falciforme em um.

No seguimento dos pacientes, $21(16,7 \%)$ necessitaram reoperação.

Dos 21 pacientes reperados, em 17 (81\%) por disfunçāo de prótese, em 16 secundário à calcificação da bioprótese e em um por escape paravalvar (4 meses após a operação).

Em quatro pacientes a reoperaçāo foi conseqüente a disfunçāo de plástica $(36 \%)$, em um foi possivel a re-plástica (dois anos e um mês após a operaçāo) e em três foi feita troca valvar (20 dias, três meses e três meses após a operaçāo).

O tempo médio de calcificação das biopróteses foi de 40 meses (3,3 anos); năo houve diferença entre as próteses porcinas e as de pericárdio bovino, com tempo médio de calcificaçāo de 32,5 meses e 32,3 meses, respectivamente (Tabela 3 ).

Nas reoperaçōes conseqüentes a calcificação das biopróteses, foram utilizadas $13(68 \%)$ próteses biológicas e $6(31 \%)$ próteses mecânicas.

As proteses mecânicas foram usadas principalmente nas reoperaçōes e os pacientes foram mantidos anticoagulados com atividade de protombina ao redor de $30 \%$; não houve fenômenos tromboembólicos no período analisado.

A mortalidade tardia foi de $2,2 \%$ (3 casos), todos por insuficiência cardíaca grave.

Com uma evolução de 2787 meses/paciente, todos encontram-se em classe funcional I e II (NYHA) (Gráfico 2).

\section{COMENTÁRIOS}

As lesōes congênitas ocorreram, principalmente, nas valvas aórtica, pulmonar e tricúspide isoladamente, sendo possível a conservação na maioria dos pacientes, $72 \%$ nos pacientes aórticos e $96 \%$ nos pacientes com lesāo da valva pulmonar. A valva tricúspide, no entanto, foi trocada em $60 \%$ dos casos e, nos pacientes com lesāo mitral congênita, a sua conservaçāo foi possível em $60 \%$ dos casos.

As lesōes na valva mitral, isoladas ou em associação, foram, principalmente, da etiologia adquirida. Nas lesōes isoladas da mitral a conservaçāo foi possível em $53,3 \%$ à medida em que ocorreu associaçāo com lesōes em outras valvas, sua conservaçāo foi menos freqüente, assim também nos mitrotricuspideos $25 \%$ e nos mitro-aórtico-tricuspídeos nos quais não foi possível a preservaçāo valvar.

A valva aórtica raramente surgiu com lesão reumática isolada; quando presente, ocorreu em associaçāo com lesão valvar mitral, sendo conservada em $37 \%$ dos casos.

A lesão valvar tricúspide isolada apresentou etiologia adquirida representada pela endocardite infecciosa, sendo necessária a remoção valvar. Quando em associçāo com a lesāo mitral, sua conservaçāo foi possível em todos os casos pela realização da plástica de De Vega.

A cirurgia valvar em pacientes jovens vem sendo um tema amplamente discutido na literatura. Seguramente, a melhor alternativa cirúrgica é a conservação valvar, que pode ser realizada através de vários procedimentos ${ }^{5 \cdot 7,36}$.

TABELA 3

PRÓTESES UTILIZADAS E TEMPO DE DURAÇĀO

\begin{tabular}{lll}
\hline Operação & Reoperação & $A T$ (meses) \\
\hline Dura-máter & Biocor & 37 meses \\
Dura-máter & Biocor & 75 meses \\
P.B. & P.B. & 24 meses \\
Biocor & P.B. & 20 meses \\
Biocor & Biocor & 51 meses \\
Biocor & P.B. & \\
Biocor & Omnicarbon & 9 meses \\
Biocor & Omnicarbon & \\
Barone & P.B. & 28 meses \\
Barone & Omnicarbon & 29 meses \\
Dura-máter & Biocor & 50 meses \\
PB & PB & 39 meses \\
Biocor & Biocor & 60 meses \\
PB & PB & 24 meses \\
PB & PB & 60 meses \\
PB & Starr-Edwards & 27 meses \\
Biocor & PB & 32 meses \\
Biocor & Omnicarbon & \\
Biocor & Omnicarbon & 20 meses \\
\hline
\end{tabular}

Média de duraçáo $=40$ meses $(3,3$ anos); bioprótese porcina 32.5 meses: bioprótese de pericárdio bovino 32,3 meses. 
GEROLA, L. R.; POMERANTZEFF, P. M. A.; PÊGO-FERNANDES, P. M.; STOLF, N. A. G.; BARBERO-MARCIAL, M.; EBAID, M.; SNITCOWSKY, R.; GRINBERG, M.; VERGINELLI, G.; JATENE, A. D. - Cirurgia valvar em crianças e jovens: resultados de 131 casos. Rev. Bras. Cir. Cardiovasc., 5(3): 187-194, 1990.

CHAUVAUD et alii ${ }^{8}$ apresentam uma série de 89 pacientes com insuficiência mitral adquirida e isolada, com idade variando de dois a 12 anos, onde a conservação da valva mitral foi possível em $92 \%$ dos casos.

A substituiçāo valvar na criança apresenta uma série de particularidades, principalmente com relação ao tipo de prótese a ser escolhida, se biológica ou mecânica.

As próteses biológicas surgiram na década de 70 e vários tecidos foram utilizados: dura-máter ${ }^{26}$, valvas homólogas ${ }^{16}$, valvas de porco e de pericárdio bovino. Atualmente, as mais utilizadas săo de pericárdio bovino e as aórticas são de porco, ambas conservadas em glutaraldeído.

Em nosso material, utilizamos, principalmente, as próteses biológicas; houve $29,3 \%$ de calcificação em um período médio de 3,3 anos.

As próteses biológicas, quando começaram a ser empragadas em pacientes pediátricos, foram aceitas com grande entusiasmo, uma vez que a incidência de tromboembolismo era baixa e não havia necessidade do uso de anticoagulante ${ }^{28,39}$. SMITH et alii ${ }^{34}$ descrevem sua experiência com 26 pacientes com menos de 19 anos submetidos a troca valvar aórtica e comparam seis tipos de próteses utilizadas: Smelloff-Cutter, Starr-Edwards, Cooley-Cutter, Hancok Porcine, Ionescu-Shiley e Björk-Shiley, e concluem ser a prótese de lonescuShiley (bovina) e de escolha do grupo, pelas características hemodinâmicas e baixa incidência de complicaçōes embólicas. GALIOTO et alii ${ }^{13}$ relatam bons resultados com o uso de próteses porcinas (Hancok) e ausência de degeneração em um período de 30 meses e preferem esta às próteses mecânicas, para pacientes jovens. No entanto, com o aumento do período de experiência, pôde-se observar a incidência precoce de calcificação e degeneraçăo do tecido ${ }^{24}$. GEHA et alii ${ }^{15}$, em avaliaçāo multicêntrica, demonstraram que $20 \%$ das valvas porcinas foram trocadas após um período médio de 33 meses de seguimento. SILVER et alii ${ }^{33}$ relataram calcificaçāo precoce ( $<4$ anos) das valvas porcinas, desaconselhando o uso delas em crianças. KOPF et alii ${ }^{20}$ descreveram $93 \%$ de calcificação e necessidade de reoperação em próteses biológicas em posiçāo aórtica, em período médio de quatro anos, e $86 \%$ de calcificaçāo e retroca das próteses biológicas em posiçāo mitral, após período médio de 3,1 anos.

Nāo estāo bem definidas as razōes pelas quais ocorre calcificaçăo precoce em pacientes jovens, bem como a melhor a durabilidade das próteses porcinas quando utilizadas em tubos extracardía$\cos ^{2,9,29}$.
Tem sido aventada uma possível reação imunológica contra a valva porcina preservada em glutaraldeído, através de estímulo antigênico e produção de anticorpos. Além disso, relacionou-se a calcificaçāo com achado de elevados níveis de ácido gama-carboxiglutâmico (GLA), um amino-ácido fixador de cálcio, em próteses porcinas calcificadas, e năo encontrado em próteses aórticas de porco recém retiradas; esse amino-ácido seria produzido pelas células envolvidas na reaçāo imunólogica, e sua concentração pode estar relacionada com o grau de infiltraçāo celular na prótese. Assim, os tubos extracardíacos ofereciam proteçāo mecânica contra essa infiltraçāo celular, o que pode explicar sua maior durabilidade 21, 27, 29. SILVER et alii ${ }^{33}$ relacionam a calcificação precoce com a hemostase do cálcio e o maior turnover de cálcio na criança.

Com o reconhecimento da calcificação precoce das próteses biológicas, as próteses mecânicas passaram a ser novamente um alternativa para os pacientes jovens. A despeito das dificuldades de anticoagulaçāo, maior susceptibilidade do jovem ao trauma, os fenômenos tromboembólicos e/ou hemorrágicos são apresentados, na literatura, com bons resultados clínicos e baixa incidência de tromboembolismo ${ }^{30,32}$.

VOUHÉ et alii ${ }^{41}$ comparam a utilizaçāo de próteses biológicas com próteses mecânicas em 155 pacientes jovens, e comentam a baixa incidência de tromboembolismo (4,0\% paciente/ano) para a valva mitral e $0,7 \%$ paciente/ano para a valva aórtica. Concluem que as próteses biológicas nāo devem ser usadas em crianças, pela elevada calcificaçāo precoce $(11 \pm 2,2 \%$ pacientes/ano)

JOHN et alii ${ }^{19}$ analisaram 118 pacientes jovens submetidos a troca valvar utilizando prótese de StarrEdwards. Em período que variou de um a 15 anos, mostraram a baixa incidência de tromboembolismo $(2,8 \%)$ e o relato de três óbitos por fenômenos hemorrágicos, devido ao anticoagulante.

ATTIE et alli ${ }^{1}$ comparam o uso de próteses mecânicas e biológicas )porcinas e dura-máter) em 110 crianças. Nas próteses mecânicas encontraram $1,7 \%$ paciente/ano de fenômenos tromboembólicos, $0,85 \%$ paciente/ano de endocardite, $0,85 \%$ paciente/ano de disfunçāo valvar e $1,3 \%$ paciente/ano de mortalidade. Nas porcinas, nāo houve fenômenos tromboembólicos, houve $2,3 \%$ paciente/ano de endocardite, 16,3 paciente/ano de disfunçāo valvar e $14 \%$ paciente/ano de mortalidade. Nas próteses de dura-máter, houve $2,3 \%$ paciente/ano de fenômenos tromboembólicos, $6,8 \%$ paciente/ano de disfunção valvar e 2,3\% paciente/ano de mortalidade.

A maioria dos trabalhos de literatura propōe a utilizaçāo de anticoagulantes orais ${ }^{3.10}$ nas próteses mecânicas. 
GEROLA, L. R.; POMERANTZEFF, P. M. A.; PÊGO-FERNANDES, P. M.; STOLF, N. A. G.; BARBERO-MARCIAL, M.; EBAID, M.; SNITCOWSKY, R.; GRINBERG, M.; VERGINELLI, G.; JATENE, A. D. - Cirurgia valvar em crianças e jovens: resultados de 131 casos. Rev. Bras. Cir. Cardiovasc., 5(3): 187-194, 1990.

GODOY et alii ${ }^{17}$ utilizaram próteses mecânicas (Lillehei-Kaster) em crianças sem o uso de anticoagulantes e relatam uma incidência de $10,7 \%$ de fenômenos trombóticos; concluem pela necessidade do uso indefinido dessa medicação. No entanto, o uso de próteses mecânicas sem o uso de anticoagulantes vem sendo aventado e tem sido sugerido o uso de antiagregantes plaquetários nesses pacientes 40 .

PASS et alii ${ }^{25}$ apresentam seus resultados com a prótese de St. Jude colocada em 34 crianças, em diversas posiçōes valvares, sem uso de anticoagulantes. Nāo foram constatados fenômenos tromboembólicos no pós-operatório em seguimento de um a 50 meses; ressaltam a necessidade de o paciente encontrar-se em ritmo sinusal, em posição mitral; quando em fibrilação atrial, utilizam anticoagulante.

VERRIER et alii ${ }^{40}$ descrevem seus resultados em 51 pacientes com idade média de 12 anos, submetidos a troca valvar aórtica por prótese mecânica (Bjöork-Shiley), usando aspirina ou aspirina e dipiridamol no pós-operatório. Não houve fenômenos tromboembólicos em seguimento que variou de três a 100 meses.

No início da década de 70, BERRY et alii ${ }^{3}$ relataram $27 \%$ de mortalidade hospitalar, STANTON et alii ${ }^{35} 25 \%$. TEIXEIRA NETO et alii ${ }^{37} 22 \%$ e MATHEW et alii ${ }^{22} 25 \%$.

A mortalidade hospitalar vem diminuindo progressivamente nos últimos anos, com a melhora técnica operatória, assistência pós-operatória e indicação precoce.

Nossa mortalidade hospitalar foi de $7,5 \%$, semelhante à encontrada nos últimos trabalhos da literatura, que varia de $2,3 \%$ a $14 \%, 1,4,8,12,40,42$.

RBCCV 44205-124

GEROLA, L. R.; POMERANTZEFF, P. M. A.; PÊGO-FERNANDES, P. M.; STOLF, N. A. G.; BARBEROMARCIAL, M.; EBAID, M.; SNITCOWSKY, R.; GRINBERG, M.; VERGINELLI, G.; JATENE, A. D. Heart valve surgery in children and adolescents. Rev. Bras. Cir. Cardiovasc., 5(3): 187-194, 1990.

ABSTRACT: From January 1983 to December 1988, 131 patients with ages iqual or less than 15 years old were submitted to valve surgery; $74(56 \%)$ were of acquired ethiology and $57(44 \%)$ were congenital. In $100(76.3 \%)$ patients operation was performed in only one valve. There were 63 valve replacements (re-replacement in six), 103 conservative procedures and in three valve exereses was performed. Among the 63 prosthesis, $59(93.6 \%)$ were biological and $4(6.3 \%)$ mechanical. Twenty-one patients $(16 \%)$ were reoperated upon, $17(81 \%)$ of them due to prosthesis dysfunction. The average time of calcification was 40 months ( 3.3 years). Hospitaly mortality was $7.5 \%$ and late mortality was $2.2 \%$. In the follow-up of 2787 months/patient all of them were in fuctional class I or II (NYHA).

DESCRIPTORS: heart valves, surgery, children; heart valves, surgery, adolescent patients.

\section{REFERÊNCIAS BIBLIOGRÁFICAS}

ATTIE, F.; KURI, J.; ZANONIANI, C.; REUTENIO, V.; BUENDIO, A.; OVSEYEVITZ, J.; LOPES-SORIANO, F.; GARCIA, C. M.; MARTINEZ-RIOS, M. A. - Mitral valve replacement in children with rheumatic heart disease. Circulation, 64: 812-815, 1981.

2 BAYLEY, W. W.; KIRKLIN, J. W.; BARGERON, L. M.; PACÍFICO, A. D.; KOUCHOUKOS, N. T. - Late results with synthetic valves external conducts from venous ventricle to pulmonary arteries. Cardiovasc. Surg., 56: $73-78,1977$.

3 BERRY, B. E.; RITTER, D. G.; WALLACE, R. B.; McGOON, D. C.; DANIELSON, D. K. - Cardiac valve replacement in children. J. Thorac. Cardiovasc. Surg., 68: 705710,1974
BLOODWELL, R. D.; HALLARAN, G. L.; COOLEY, D. A. Cardiac valve replacement in children. Surgery, 63: $77-89,1968$.

5 CARPENTIER, A. - Cardiac valve surgery: the french corretion. J. Thorac. Cardiovasc. Surg., 86: 323$337,1983$.

6 CARPENTIER, A.; CHAUVAUD, S.; FABIANI, J. M.; DELOCHE, A.; RELLAND, J.; LESSANA, A.; D'ALLAINES, C.; BLONDEAU, P.; PIWNICA, A.; DUBOST, C. - Reconstrutive surgery of mitral valve incompetence: ten-years appraisal. J. Thorac. Cardiovasc. Surg., 79: 338-348, 1980.

7 CARPENTIER, A.; CHAUVAUD, S.; NOSÉ, L.; RELLAND, J.; MICHILEANU, S.; MARINO, J. P.; ABRY, B.; GUIBOURT, P. - A new reconstrutive operation for 
GEROLA, L. R.; POMERANTZEFF, P. M. A.; PÊGO-FERNANDES, P. M.; STOLF, N. A. G.; BARBERO-MARCIAL, M.; EBAID, M.; SNITCOWSKY, R.; GRINBERG, M.; VERGINELLI, G.; JATENE, A. D. - Cirurgia valvar em crianças e jovens: resultados de 131 casos. Rev. Bras. Cir. Cardiovasc., 5(3): 187-194, 1990.

Ebstein anomaly of the tricuspid valve. J. Thorac. Cardiovasc. Surg., 96: 92-101, 1988.

CHAUVAUD, S.; PIRIER, P.; TONATI, G.; RELLAND, J.; KARA, S. M.; CARPENTIER, A. - Long term results of valve repair in children with acquired mitral valve incompetence. Circulation, 74 (Supl. 1): 104109, 1986.

CIAROVELLA, J. M.; McGOON, D. C.; DANIELSON, G. K.; WALLACE, L. B.; MAIR, D. C. - Experience with the extracardiac conduit. J. Thorac. Cardiovasc. Surg., 78: 920-930, 1979.

CHEN, S.; LAKS, H.; FOGAN, C.; RERSCHLUSE, D.; KAISER, G.; BARNER, H. H.; WILLIMAN, V. L. Valve replacement in children. Circulation, 56 (Supl. 2): 117-121, 1977.

DUNN, J. M.; - Porcine valve durability in children. Ann. Thorac. Surg., 32: 357-368, 1981.

12 ELOY, R.; FERREIRA, A.; CARVALHO, H.; BRITO, J. C.; TADEU, E.; NERY, A. C.; RIBEIRO, A.; OLIVEIRA, R.; RIBEIRO, N. - Cirurgia orovalvar na criança: experiência de 135 casos. Rev. Bras. Cir. Cardiovasc., 1: 24-28, 1986.

GALIOTO, F. M.; MIGDLEY, F. M.; SHAPIRO, S. P.; PERRY, L. W.; CHAVARELLA, J. M.; SCOTT, L. P. - Mitral valve replacement in infants and children. Pediatrics, 67: 230-235, 1981

GARDNER, T. J.; ROLAND, J. M. A.; NEILLE, C. A.; DONAHHOO, J. S. - Valve replacement in children: a fifteen years perspective. J. Thorac. Cardiovasc. Surg., 83: 178-185, 1982.

GEHA, A. S.; LAKS, A.; STANSEL Jr., H. C.; CORNHILL, J. F.; KILMAN, J. N.; BUCKLEY, M. J.; ROBERTS, W. C. - Late failure of porcine valve heterografts in children. J. Thorac. Cardiovasc. Surg., 78: 351 $364,1979$.

GIMENES, A. C.; BUFFOLO, E.; FORTE, V.; ANDRADE, J. S. C.; GOODY, M.; SUCCI, J. E.; GIMENES, V. M.; CURY, A.; GALLUCI, C. - Resultados tardios (mais que 5 anos) de pacientes portadores de valva homóloga montada em suporte na substituiçāo de valvas cardíacas. Rev. Bras. Med., 34: 48-52, 1976.

GODOY, M. F.; BRANCO, J. N; SOARES, H. C.; LEĀO, L. E. V.; CARVALHO, A. C. C.; GIMENEZ, A. C.; RIBEIRO, E.; SUCCI, J. E.; ANDRADE, J. C.; ALBERTINS M. V.; VENTRIGLIA, C. R. B.; BUFFOLO, E.; GALLUCCI, C. - Relato a longo prazo da substituição valvar em crianças. Arq. Brasil. Cardiol. 37: 325-329, 1981.

HUMAN, D. G.; JOFFE, H. S.; FRASER, C. B. - Mitral valve replacement in children. J. Thorac. Cardiovasc. Surg., 83: 873-877, 1982. S.; RAVIKUMAR, I. P. - Mitral valve replacement in

the young patient with rheumatic heart disease. J. Thorac. Cardiovasc. Surg., 86: 209-216, 1983.

KOPF, G. S.; GEHA, A. S.; HELLENBRAND, W. E.; KLEINMAN, C. S. - Fate of left-side cardiac bioprosthesis valves in children. Arch. Surg., 121: 488-490, 1986.

LEVY, R. J.; ZENKER, J. A.; LION, J. B. - Vitamin kdepleted calcium binding proteins in aortic valve calcification. J. Clinic. Invest., 65: 563-566, 1980.

MATHEW, R. A.; PARK, S. C.; NECHES, D. H.; LENOX, C. C.; ZUBERBUHLER, J. R.; LERBERG, D. B.; BAHNSON, H. T. - Valve replacement in children and adolescent. J. Thorac. Cardiovasc. Surg., 73: 872-878, 1977.

MILANO, A., BORTOLOTTI, V.; TALENTE, E.; VALFRÈ, C.; ARBUSTINI, E.; VALENTE, M.; MAZZUCO, A.; GALLUCI, V.; THIENE, G. - Calcific degeneration as the main cause of porcine bioprosthetic failure. Am. J. Cardiol., 53: 1066-1070, 1984.

24 MILLER, D. C.; STINSON, E. B.; OYER, P. E.; BILLINGHAN, M. E.; PITLICK, P. T.; REITZ, B. A.; JAMIESON, S. W.; BAUMGARTNER, W. A.; SHUMWAY, N. E. - The durability of porcine xenograft valves and conduct in children. Circulation, 66: $172-185,1982$.

PASS, H. I.; SADE, R. M.; CRAWFORD, F. A.; HOLM, A. - Cardiac valve prosthesis in children without anticoagulation. J. Thorac. Cardiovasc. Surg., 87: $832-835,1984$.

PUIG, L. B.; VERGINELLI, G.; SOSA, E. A.; ROMA, L. S. L.; GARCIA, D.; CONCEIÇĀO, A. N. - Avaliação hemodinâmica da valva de dura-máter mitral e tricúspide. Arq. Bras. Cardiol.; 29: 297-301, 1976.

27 ROCCHINI, A. P.; WEESNER, K. M.; HEIDELBERG, K.; KEREN, D.; BEHRENDT, B.; ROSENTHAL, A. Porcine xenograft valve failure in children: an immunologic response. Circulation, 64 (Supl. 2): $162-171,1981$.

28 RUPPRATH, G.; FRANKE, F.; VOGT, J.; De VIVIE, E. R. - Long term results after mitral and aortic valve replacement childhoold and adolescence. Z. Kardiol., 75 (Supl. 2): 312-317, 1986.

RUPPRATH, V. J.; De VIVIE, E. R.; BEUREN, A. J. Conduit repair for complex congetinal heart disease with pulmonary atresia or right ventricular outflow tract obstruction. Part II: Early and late hemodynamic and echocardiographic findings. J. Thorac. Cardiovasc. Surg., 29: 337-341, 1981.

SADE, R. M.; BALLENGER, J. F.; HEHN, A. R.; QUANTS J. E.; RIOPEL, D. A.; TAYLOR, A. B. - Cardiac valve replacement in children: comparison of tissue with mechanical prothesis. J. Thorac. Cardiovasc. Surg., 78: $123-127,1979$

31 SANDER, S. P.; LEVY, R. J.; FREED, M. B.; NORWOOD, 
GEROLA, L. R.; POMERANTZEFF, P. M. A.; PÊGO-FERNANDES, P. M.; STOLF, N. A. G.; BARBERO-MARCIAL, M.; EBAID, M.; SNITCOWSKY, R.; GRINBERG, M.; VERGINELLI, G.; JATENE, A. D. - Cirurgia valvar em crianças e jovens: resultados de 131 casos. Rev. Bras. Cir. Cardiovasc., 5(3): 187-194, 1990.

D. I.; CASTAÑEDA, A. R. - Use of Hancock porcine xenografts in children and adolescents. Am. J. Cardiol., 46: 429-438, 1980.

SCHACHNER, A.; SALOMON, J.; LEON, L.; LEONARD, C. B.; LEVY M. J. - Prosthetic valve replacement in infants and children. J. Thorac. Cardiovasc. Surg., 25: $537-544,1984$.

SILVER, M. M.; PALLOCKF, J. S.; WILLIAMS, W. G.; TRUSLER, G. A. - Calcification in porcine xenograft valves in children. Am. J. Cardiol., 45: 685-689, 1980.

SMITH, J. M.; COOLEY, D. A.; OTT, D. A.; FERREIRA, W.; RUEL Jr., G. A. - Aortic valve replacement in children. Ann. Thorac. Surg. 29: 512-518, 1980.

STANTON, R. E.; GALLAGHER, M. E.; MEYER, B. W.; LINDESMITH, G. G.; STILES, A. R. - Replacement of cardiac valves in children. Am. J. Dis. Child. 120: $503-510,1970$.

SULAYAMAN, R.; MATTHEW, R.; HICENIUS, O. G.; REPLAGE, R.; ARCILLA, R. A. - Hemodynamics and annuloplasty in isolated mitral regurgitation in children. Circulation, 52: 1144-1155, 1975.

37

TEXEIRA NETO, J. A.; STOLF, N. A. G.; PUIG, L. B.; SOSA, E.; PILEGGI, F.; BITTENCOURT, D.; VERGINELLI, G.; ZERBINI, E. J. - Substituiçāo de valvas cardíacas por próteses em crianças e pa- cientes jovens. Arq. Brasil. Cardiol., 28: 409-415, 1975.

38 THADROYEN, F. T.; WILTON, I. N.; PIRIE, D.; ROGERS, M. A.; MITHA, A. S. - Severe calcification of glutaraldehyde preserved porcine xenografts in children. Am. J. Cardiol., 45: 690-696, 1980.

39 THIENE, G.; BORTOLOTTI, V.; PANIZZON, C.; MILANO, A.; GALUCCI, V. - Pathological substrates of thrombus formation after heart valve replacement with the Hancock bioprosthesis. J. Thorac. Cardiovasc. Surg., 80: 414-423, 1980.

40 VERRIER, E. D.; GRANBAUGH, R. F.; SAIFER, S. J.; YEE, E. S.; TURLEY, K.; EBERT, P. A. - Aspirin anticoagulation in children with mechanical aortic valves. J. Thorac. Cardiovasc. Surg., 92: 10131020, 1986.

41 VOUHÉ, P. R.; MILANO, A.; LECO, F.; NEVEUX, J. Y. - Aortic and mitral valve replacement in young patients Z. Kardiol., 75 (Supl. 2): 289-293 - 1986.

42 WEINSTEIN, G. S.; MARROUDIS, C.; EBERT, P. A. Preliminary experiences with aspirin anticoagulation in children with prosthetic cardiac valves. Ann. Thorac. Surg., 33: 549-554, 1982.

43 WILLIAMS, D. B.; DANIELSON, G. K.; McGOON, D. C.; PUGA, F. J.; MAIR, D. D.; EDWARDS, W, D. Porcine heterograft valve replacement in children. J. Thorac. Cardiovasc. Surg., 84: 446-450, 1982. 